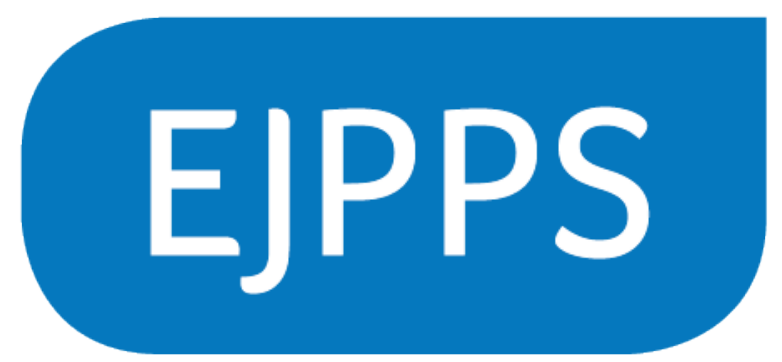

EUROPEAN JOURNAL OF

PARENTERAL AND

PHARMACEUTICAL SCIENCES

EJPPS - European Journal of Parenteral and Pharmaceutical Sciences Volume 26 Issue 4

https://www.ejpps.online/post/vol26-4-end-to-end-qualification-of-ready-to-use-rtu-productcontainers-in-packaging-suitable\#viewer-cltu5

https://doi.org/10.37521/ejpps.26401

Title: End-to-end qualification of ready-to-use (RTU) product containers in packaging suitable for No-Touch Transfer (NTT) into Grade A filling zones.

Birte Scharf ${ }^{1}$ Patrick Wolf ${ }^{2}$ Holger Kranenburg ${ }^{3}$ and Robert Lindner ${ }^{4}$

1) Birte Scharf, Franz Ziel GmbH

2) Patrick Wolf, SCHOTT AG

3) Holger Kranenburg, Franz Ziel GmbH

4) Robert Lindner, SCHOTT AG

Corresponding Author: Holger Kranenburg

Franz Ziel GmbH

Raiffeisenstr. 33

Billerbeck 48727

Germany

Email: holger.kranenburg@ziel-gmbh.com 


\title{
Title: End-to-end qualification of ready-to-use (RTU) product containers in packaging suitable for No-Touch Transfer (NTT) into Grade A filling zones.
}

\author{
Birte Scharf ${ }^{1}$ Patrick Wolf ${ }^{2}$ Holger Kranenburg ${ }^{3}$ and Robert Lindner ${ }^{4}$
}

1) Birte Scharf, Franz Ziel GmbH

2) Patrick Wolf, SCHOTT AG

3) Holger Kranenburg, Franz Ziel GmbH

4) Robert Lindner, SCHOTT AG

\begin{abstract}
No-Touch Transfer (NTT) of pre-sterilised ready-to-use (RTU) containers is an alternative methodology that follows Good Manufacturing Practice (GMP) and Quality Risk Management (QRM) principles. NTT de-bagging ejects contents from secondary bag packaging without direct contact with contents or exposure to an environment that is a lower grade than the zone being entered. The pre-sterilised containers and sterile barriers offer assured sterility at manufacture and are qualified to remain sterile through the supply chain and the stepwise NTT de-bagging process. This eliminates the requirement for in-process material disinfection steps for transfer into Grade A environments.

The present article focuses on design qualification of pre-sterilised RTU container packaging, including definition of sterile barriers together with bioburden study data through the supply chain and simulated NTT. It completes a series of EJPPS articles to support peer-reviewed references on NTT. Together, these articles can be defined as end-to-end qualification of the NTT process, demonstrating a high level of assurance that sterility is maintained from manufacture to point of use.
\end{abstract}

Key Words: Aseptic processing, Design qualification, Good Manufacturing Practice (GMP), Life cycle, No-Touch Transfer (NTT), Pharmaceutical packaging, Pre-sterilised containers, Qualification, Quality by Design (QbD), Quality Risk Management (QRM), Ready-to-use (RTU), Supply chain 


\section{Introduction}

NTT is an alternative methodology for the transfer of RTU pre-sterilised product containers into a Grade A zone for filling sterile products. Compared to traditional manufacturing processes, which require an in-process outer packaging surface bio-decontamination step, i.e. E-beam or vaporised hydrogen peroxide $\left(\mathrm{vH}_{2} \mathrm{O}_{2} / \mathrm{VHP}\right),{ }^{1,2}$ the NTT bases its technological development on GMP as well as QRM principles.

Proof-of-concept studies and qualification of contamination control measures during the NTT process applied to a filling line barrier system have been demonstrated to successfully manage both surface and airborne contamination risks. ${ }^{3}$

Following the concept of NTT, the pre-sterilised containers enter the cleanroom facility in double bags and are transferred zone by zone by debagging without an in-process disinfection/decontamination step. Studies have verified that Grade A filling conditions are not compromised in the transfer process. ${ }^{4}$ The proof of the NTT concept and qualification of surface and airborne contamination control measures during the NTT in-process material transfers ${ }^{3}$ opened the opportunity for NTT technology development and optimisation including semi-automated and fully automated in-process transfers of RTU containerpackaging with progressive de-bagging steps en route to the Grade A processing environment. Requirements in qualification studies to follow GMP and QRM principles for the in-process transfers on an aseptic filling line were aligned through PHSS: Pharmaceutical and Healthcare Sciences Society, Aseptic Processing special interest group discussions including pharma industry key opinion leaders and Medicines and Healthcare products Regulatory Agency GMP inspectors and subsequently published in the EJPPS. ${ }^{4}$

At the same time, it is essential to qualify the suitability and processability of NTT packaging to support the assurance of sterility and that sterile barriers are not compromised through the supply chain and NTT in-process material transfers. Consequently, the packaging has to be designed based on a Quality by Design ( $Q b D$ ) approach so the pre-sterilised, nested/in-tray containers within have defined sterile barriers that maintain sterility from manufacture to use as an end-to-end approach (Figure 1). Furthermore, the packaging material has to be designed and qualified for processability on automatic de-bagging machines. ${ }^{5}$

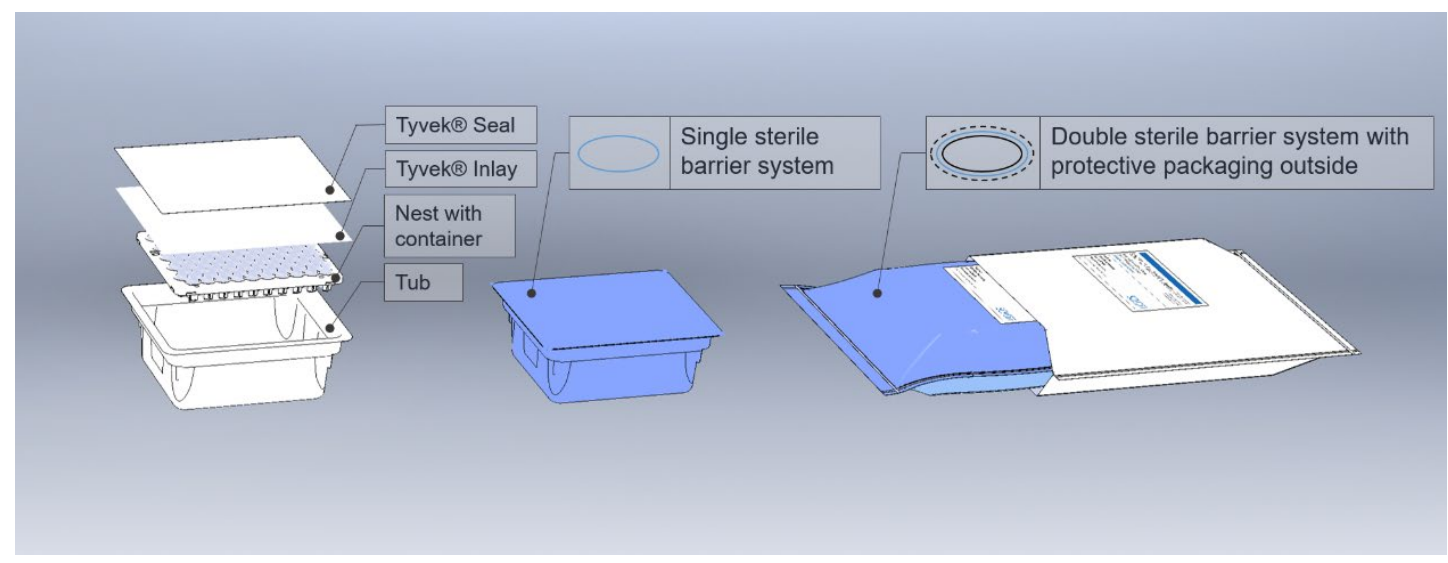

Figure 1: Schematic illustration of the pre-sterilised RTU container packaging and definition of sterile barrier systems. 
Supply chain qualification is required for NTT suitability to assure sterile barriers are not compromised by contamination in transport to manufacturing sites. If the supply chain has potential risks of damaging the packaging, consideration should be given to shock indicators that provide an alert about shocks experienced in the supply chain. ${ }^{5}$

This article focuses on design qualification of pre-sterilised RTU container packaging including definition of sterile barriers together with bioburden study data through the supply chain and simulated NTT in-process transfers into a Grade A processing environment of a barrier technology isolator.

To qualify the continuity of sterility through the supply chain, independent microbiological studies of bioburden on RTU container packaging layers were completed. As proof of the robustness and verification that sterile barriers had not been compromised through the supply chain of the proposed NTT methodology, the receipt of supplies at a user site has been simulated under worst-case conditions by preparation of the packaging assemblies under R\&D conditions. To determine bioburden on packaging layers and through layer removal with exposure of sterile barriers, a simulated NTT process was part of the study design.

\section{Material and Methods; Microbiological Profile Studies}

\subsection{Study Material}

Pre-sterilised containers (vials, syringes, cartridges) were provided by SCHOTT AG in a doubledbagged format suitable to the NTT process. Typically, ethylene oxide (ETO)/steam presterilised containers are sterilised as nest/tray formats with a sterile barrier on the tub-Tyvek ${ }^{\circ}$ level, which is only removed (de-lidded) after the introduction of the tub/tray into a Grade $A$ environment. For the SCHOTT AG NTT packaging, an additional sterile barrier was introduced on the innermost bag level and a protective outer bag. ETO-sterilised products are packaged as a composite assembly in a carton, e.g. perforated polymer cartons prior to sterilisation, while steam-sterilised products are composite-assembled into cartons after sterilisation. For delivery, cartons are stacked on pallets, overwrapped, and strapped.

For this specific study material, an atypical worst-case microbial challenge formed part of the study design to provide a high level of assurance that bioburden from outer surfaces of cartons and outer secondary packing layers, which are in contact with the inside of the perforated cartons, does not compromise the sterile barriers through the supply chain. Hence, instead of packaging the double-bagged containers within cartons in the qualified production manufacturing area, samples were prepared in an R\&D area extending the bioburden profile to include fungi and moulds, which would not be represented in a production batch supply. 


\subsection{Methods}

To provide a straightforward approach for the necessary high level of assured sterility, collective qualification studies were conducted. Collective studies include surface sampling at packaging layer removals with the expectation of 0 colony-forming units (CFU) recovery on all sterile barriers. Furthermore, bioburden studies provide evidence that surface contamination on transport cartons on receipt through a supply chain does not transfer to the sterile barriers during NTT steps. For supply chain microbial profile qualification, the sterile barriers of a representative batch of multiple RTU units in cartons should demonstrate 0 CFU recovery. The outer surfaces of the secondary packaging bags and final sterile barrier should not demonstrate microbial levels higher than the zone they are to be transferred into. In the case of the final sterile barrier, 0 CFU recovery is expected at entry to Grade A for subsequent delidding (removal of Tyvek ${ }^{\circ}$ lid-cover) and filling/closing.

\subsubsection{Design qualification of the suitability of pre-sterilised RTU product container packaging for applications of NTT in-process transfers in aseptic process filling.}

The RTU product container packaging used in this study was specifically designed to be the perfect fit for NTT suitability using a QbD approach. Besides the process understanding and control, a key factor in this approach is the product design based on quality target product profiles. Thus, critical quality attributes (CQA) and critical material attributes (CMA) are identified. ${ }^{6}$ For the NTT packaging such CQA and CMA are, for example, the integrity of the innermost bag and the sterile barrier properties of the materials specified.

Consequently, the NTT packaging solution is based on double-bagged RTU containers with double sterile barrier systems. ${ }^{7}$ The first sterile barrier being the tub/tray of nested containers with Tyvek ${ }^{\circ}$ or suitable alternative covering and the second sterile barrier being the innermost bag (Figure 1).

For the design of a sterile barrier and the design qualification, the ISO 11607-1 provides general guidance. ${ }^{7}$ An essential requirement is the demonstration of the sterile barrier properties of the materials as well as the integrity of the designed packaging by means of performance testing, e.g. dye penetration testing, bubble leak testing, etc. In addition to the initial design qualification, two other pillars are important for the specification of sterile barrier properties of the innermost bag on an end-to-end level, process validation according to the requirements provided in the ISO $11607-2^{8}$ and performance testing on sterile barrier systems after representative transportation and shelf-life conditions (Figure 2). 


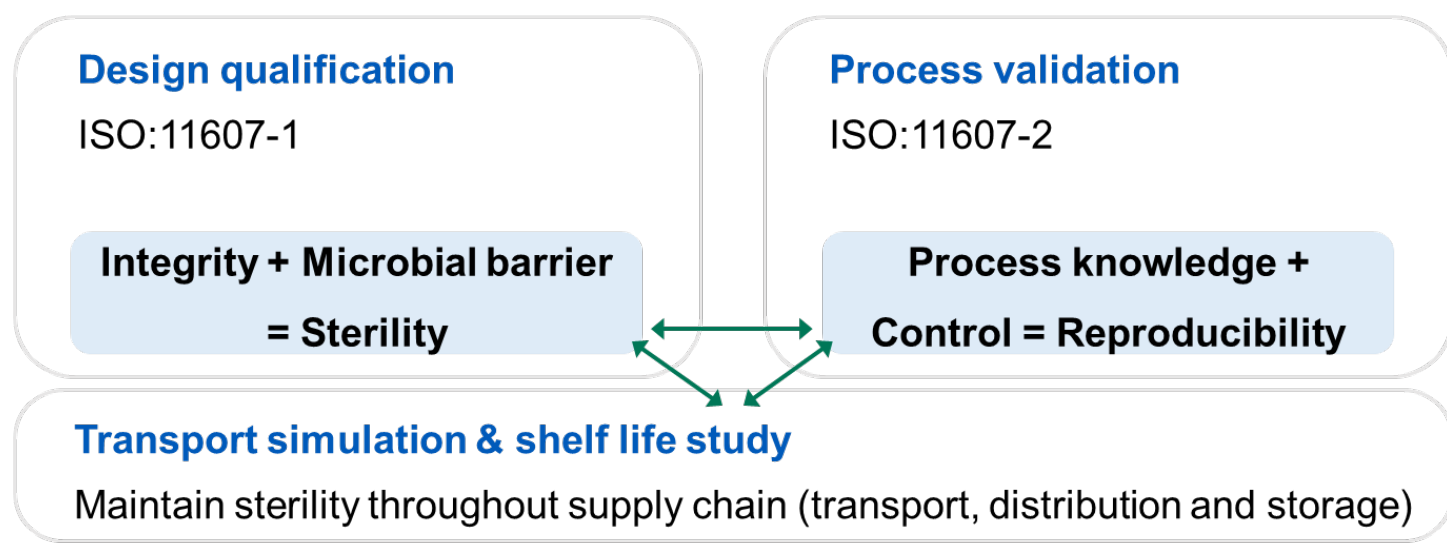

Figure 2: Three pillars for the implementation of a sterile barrier on the inner bag level. A) Design qualification including performance testing of material and design of the defined sterile barrier system in accordance to the requirements of ISO 11607-1; B) Process validation in accordance to the requirements of ISO 11607-2; and C) Representative transport simulation and shelf-life study to demonstrate the maintenance of claimed performance.

\subsubsection{Qualification study design}

The study included three independently performed steps with one carton per configuration of container types (cartriQ ${ }^{\circ}$ RTU steam-sterilised cartridges, adaptiQ ${ }^{\circ}$ ETO-sterilised RTU vials, and syriQ ${ }^{\circ}$ pre-fillable, ETO-sterilised RTU syringes, with 15 tubs in each carton).

Study execution as a simulated NTT supply chain receipt, NTT in-process transfer aseptic process simulation into a Grade $A$ isolator (subjected to qualified 6 log sporicidal $\mathrm{vH}_{2} \mathrm{O}_{2} / \mathrm{VHP}$ gaseous bio-decontamination cycle) was completed within the facility and in-house microbiology laboratory of Franz Ziel in Germany. Studies were managed by scientists and executed by qualified microbiologists and biologists with protocols prepared in GMP format to guide study procedure, execution, and result capture for review and approval.

Additional atypical challenges to contamination control and surrounding environment bioburden were included in the study design.

The microbial profile bioburden study was conducted as a process simulation of supply chain and NTT debagging steps with the extra challenges, such as the surrounding environment being controlled but not classified (CNC). In addition, lab work (rather than cleanroom) gowning was worn, including lab coat, gloves, and face mask.

For the study, NTT in-process material transfers were based on lab-manual procedures and not a fully automated or semi-automated NTT de-bagging process that is recommended as a minimum for the final NTT transfer step into a Grade A environment of a GMP operational filling line. ${ }^{4}$ 
Multiple worst-case challenges including atypical bioburden (yeasts and moulds) on sample container packaging/cartons, lab-based gowning with a non-classified background to the Grade A study isolator, and manual procedures for NTT transfer steps were combined. If the bioburden studies met the acceptance criteria of 0 CFU recovery on sterile barriers without compromise to the isolator Grade A environment in this more challenging scenario, the results would provide a much higher level of assurance that the NTT process was not operating at the edge of failure. Considering the worst case demonstrates the risk of contamination of sterile barriers and transferring contamination into the Grade A environment, thereby compromising container/product sterility when the sterile barrier Tyvek ${ }^{\circ}$ lid is removed and aseptic filling begins.

\subsubsection{Bioburden studies}

As a study starting point, the simulation of the supply chain receipt to study incoming bioburden was performed beginning with the reception of the cartons at the Franz Ziel warehouse Building 1. Two contact plate samples were taken at each side of the three carton boxes to determine the bioburden (Figure $3 \mathrm{~A}$ ). The plates were incubated at $20-25^{\circ} \mathrm{C}$ and 30 $35^{\circ} \mathrm{C}$ for five days. Afterwards, CFU were counted by trained personnel, and any recovered microorganism was identified with Gram staining and via API ${ }^{\circ}$.

The cartons were transferred to the microbiological laboratory material entry point and opened in a CNC zone. With disinfected gloves, each of the 15 double-bagged tubs were removed one by one from the carton, transferred into the lab, and placed into a unidirectional air flow (UDAF) clean bench that directly interfaced with the Grade $A$ isolator via an entry mouse hole, simulating an NTT process zone in a Grade $\mathrm{C}$ area. Both sides of the outer bagged containers were swabbed using two swabs as soon as the tub was placed on the clean bench (Figure $3 \mathrm{~B}$ ). Both swabs were streaked out on tryptic soy agar for incubation at $20-25^{\circ} \mathrm{C}$ and 30-35 C for five days and afterwards CFU were counted by trained personnel.

In the UDAF clean bench, at a specified control point that simulates a Grade C NTT zone, the outer secondary packaging bag was cut open via a manual procedure and the inner bagged contents ejected without direct touch into the second defined section of the UDAF clean bench, simulating Grade B NTT zone.

Following gloved hand disinfection procedures, both sides of the inner bag were swabbed (Figure $3 \mathrm{C}$ ), but this time, the swab was directly placed into tryptic soy broth (TSB), as it was expected to have no growth. The TSB was incubated at $30-35^{\circ} \mathrm{C}$ for five days and afterwards observed for growth (turbidity) or no growth (no turbidity). 
Simulating the NTT transfer step into Grade A (Figure 4), the inner sealed header bag was then cut open via a manual procedure and the tub was ejected through the mouse hole into the Grade A environment of the bio-decontaminated isolator without directly touching the ejected contents (sterile barrier comprising tub of containers with Tyvek ${ }^{\circ}$ lid-cover). Each side of the tub was swabbed (Figure 3D) and swabs were directly transferred into TSB inside the isolator. After eject transfer of the tub into the isolator, samples were taken from the inner header bag via contact plates. The TSB was incubated at $30-35^{\circ} \mathrm{C}$ for five days and afterwards observed for growth (turbidity) or no growth (no turbidity). The contact plates were incubated at $30-35^{\circ} \mathrm{C}$ for five days, no externally transferred contamination is expected for this layer, therefore no specific incubation temperature for yeasts and moulds is required in this step, incubation temperature is optimized for human commensals to determine transferred contamination ${ }^{9}$. Afterwards, CFU were counted by trained personnel.

The Tyvek ${ }^{\circ}$ lid was then removed and swab samples were taken from the container nest and on the edges where the Tyvek ${ }^{\circ}$ was adhered/glued on. (Figure 3E) The swabs were then directly transferred into TSB inside the isolator. The TSB was incubated at $30-35^{\circ} \mathrm{C}$ for five days and afterwards observed for growth (turbidity) or no growth (no turbidity).

To mitigate and eliminate the risk that microbial/ sporicidal contamination is held in the Tyvek ${ }^{\circ}$ filter matrix, which cannot be recovered by surface sampling with swabs or contact plates, a different type of study was completed. This study considered if any contamination from the Tyvek ${ }^{\circ}$ cover-lid could potentially pose a risk. As both sides of the filter matrix are exposed to Grade A on removal and during manipulation, there potentially could be a contamination release from the Tyvek ${ }^{\circ}$. In this study, the Tyvek ${ }^{\circ}$ lid-cover was cut into six strips for use as test samples that were directly transferred into TSB (Figure 3F). Three samples were incubated at $55-60^{\circ} \mathrm{C}$ and three at $30-35^{\circ} \mathrm{C}$ for five days and observed for growth (turbidity) or no growth (no turbidity). These temperatures were chosen to allow growth of probable contaminants in the lab environment: human commensals $\left(30-35^{\circ} \mathrm{C}\right)$ and Geobacillus stearothermophilus spores $\left(55-60^{\circ} \mathrm{C}\right)$.

In total 45 tubs, i.e. the complete content of three cartons, were tested in the described way. 


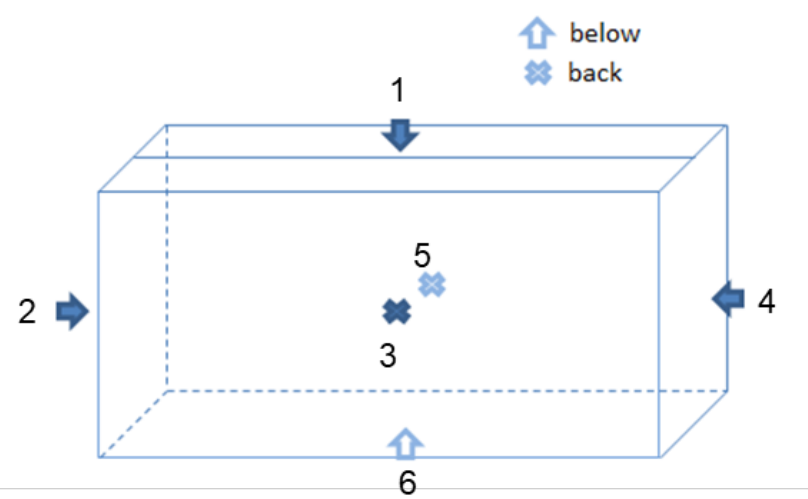

A: Schematic sample design for polymeric carton

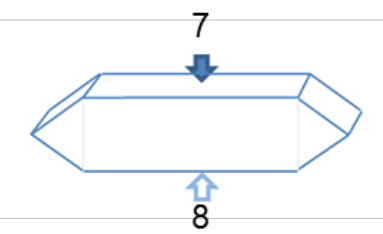

B: Schematic sample design for outer bag

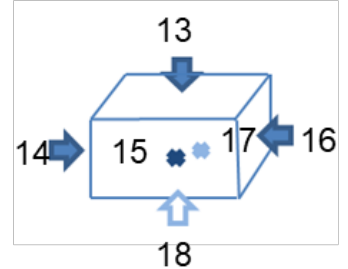

D: Schematic sample design for tub

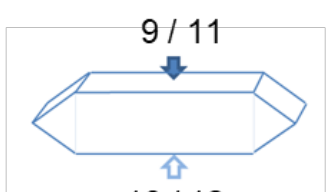

$10 / 12$

C: Schematic sample design for inner bag

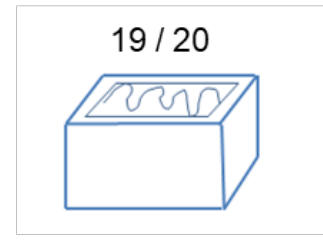

\section{E: Schematic sample design for barrel nest - stroke between} containers

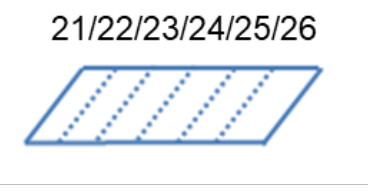

F: Schematic sample design for Tyvek cuts

Figure 3: Schematic sample design for sampling locations 1-26

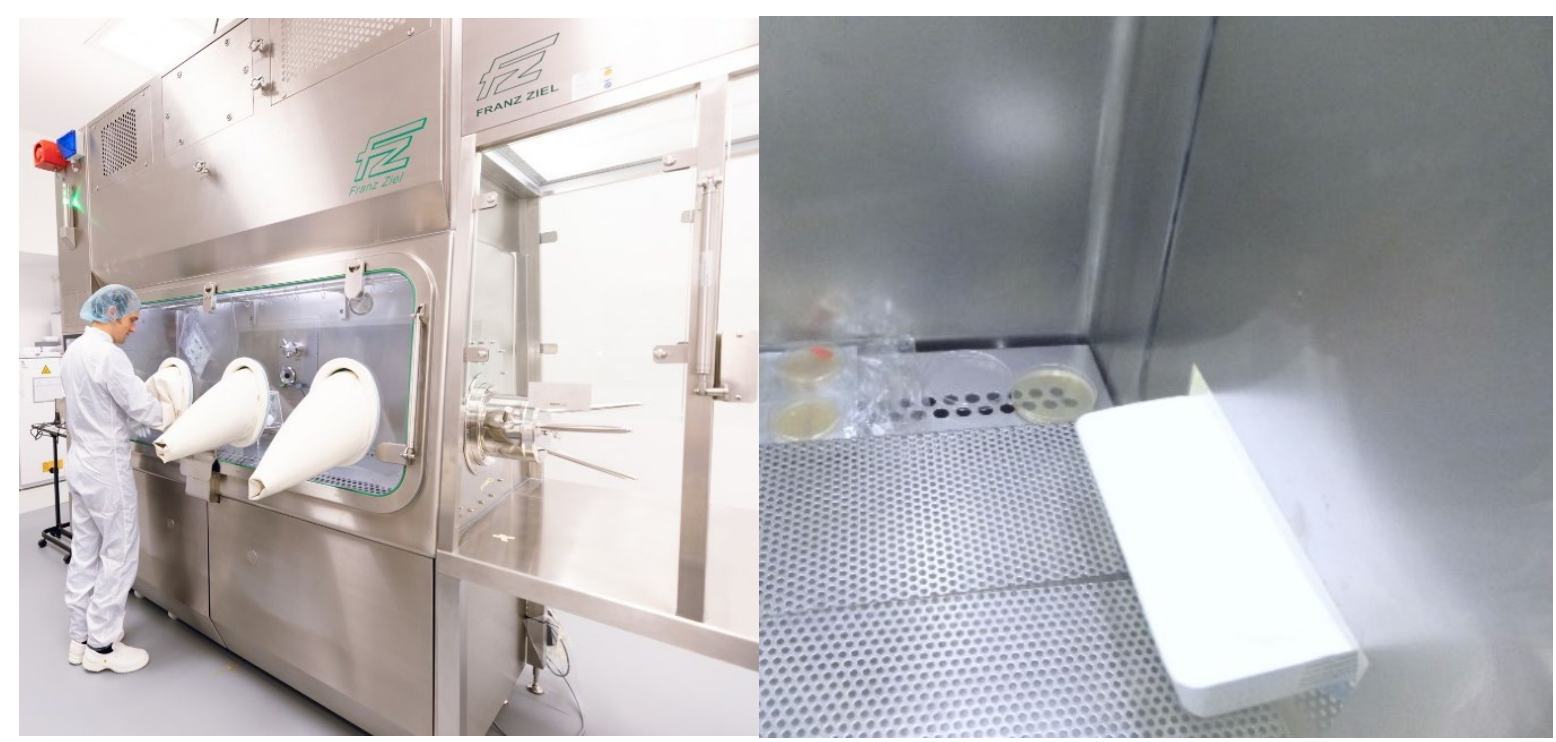

Figure 4: Picture of the isolator and UDAF clean bench. Transfer of the tub inside the isolator (Grade A). 


\section{Results}

\subsubsection{Bioburden studies}

As mentioned above, sample tubs were prepared with the worst-case microbial challenge including yeasts/moulds, which is atypical for materials supplied to the pharma industry.

Table 1: Results of bioburden studies with worst-case simulation.

\begin{tabular}{|l|l|l|l||l|}
\hline & $\begin{array}{l}\text { syriQ } \\
\text { pre-fillable, ETO- } \\
\text { sterilised RTU } \\
\text { syringes }\end{array}$ & $\begin{array}{l}\text { adaptiQ }^{\circledR} \\
\text { ETO-sterilised RTU } \\
\text { vials }\end{array}$ & $\begin{array}{l}\text { cartriQ } \\
\text { RTU steam- } \\
\text { sterilised cartridges }\end{array}$ & Acceptance criteria \\
\hline $\begin{array}{l}\text { Outer } \\
\text { carton }\end{array}$ & $\begin{array}{l}49 \text { CFU } \\
\text { Yeast, moulds, } \\
\text { bacteria (human } \\
\text { commensals) }\end{array}$ & $\begin{array}{l}\text { Yeast, moulds, } \\
\text { bacteria (human } \\
\text { commensals) }\end{array}$ & $\begin{array}{l}\text { Yeast, moulds, } \\
\text { bacteria (human } \\
\text { commensals) }\end{array}$ & $\begin{array}{l}\text { No limit (bioburden } \\
\text { determination) }\end{array}$ \\
\hline $\begin{array}{l}\text { Outer } \\
\text { bag } \\
\text { outside }\end{array}$ & $\begin{array}{l}3 \text { CFU total on } 15 \\
\text { bags } \\
1 \text { CFU mould }\end{array}$ & $\begin{array}{l}24 \text { CFU total on 15 } \\
\text { bags } \\
9 \text { CFU mould }\end{array}$ & $\begin{array}{l}89 \text { CFU total on 15 } \\
\text { bags } \\
14 \text { CFU mould }\end{array}$ & $\begin{array}{l}\text { Not to exceed levels } \\
\text { in the surrounding } \\
\text { environment }\end{array}$ \\
\hline $\begin{array}{l}\text { Inner bag } \\
\text { outside }\end{array}$ & $\begin{array}{l}\text { Swabs: no growth } \\
\text { contact: } 3 \text { CFU total } \\
\text { on } 15 \text { bags }\end{array}$ & $\begin{array}{l}\text { Swabs: no growth } \\
\text { contact: 7 CFU total } \\
\text { on 15 bags }\end{array}$ & $\begin{array}{l}\text { Swabs: no growth } \\
\text { contact: 12 CFU } \\
\text { total on 15 bags }\end{array}$ & $\begin{array}{l}\text { Swabs: no growth } \\
\text { surface sampling: } 5 \\
\text { CFU per bag }\end{array}$ \\
\hline Tub & No growth & No growth & No growth & No growth \\
\hline Tyvek & No growth & No growth & No growth & No growth \\
\hline
\end{tabular}

Table 2: Examples for bioburden reduction with worst-case simulation.

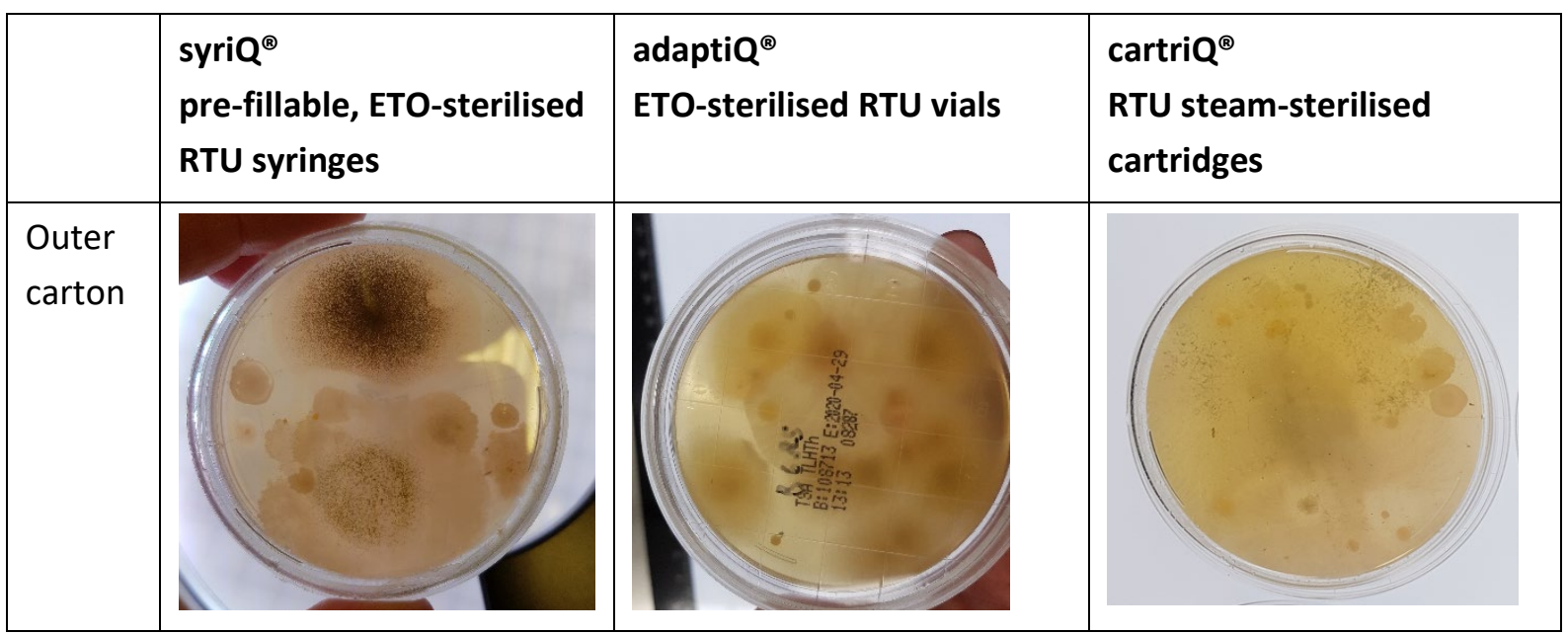




\begin{tabular}{|l|l|l|l|}
\hline Tub & \\
& & & \\
(clear - no turbidity) & (clear - no turbidity) & (clear - no turbidity) \\
\hline
\end{tabular}

Table 3: Results of Environmental monitoring during tests (samples were taken in the cleaner controlled areas of the laboratory). Settle plates exposed throughout the testing procedure ( $<4$ hours) for each of three container types.

\begin{tabular}{|l|l|l|l|}
\hline & $\begin{array}{l}\text { syriQ } \\
\text { pre-fillable, ETO- } \\
\text { sterilised RTU syringes }\end{array}$ & $\begin{array}{l}\text { adaptiQ } \\
\text { ETO-sterilised RTU } \\
\text { vials }\end{array}$ & $\begin{array}{l}\text { CartriQ }^{\circledR} \\
\text { RTU steam- } \\
\text { sterilised cartridges }\end{array}$ \\
\hline $\begin{array}{l}\text { Laboratory (CNC) } \\
\text { settle plate }\end{array}$ & 5 CFU & 5 CFU & 8 CFU \\
\hline $\begin{array}{l}\text { UDAF unit Part C } \\
\text { settle plate }\end{array}$ & 1 CFU & 2 CFU & 3 CFU \\
\hline $\begin{array}{l}\text { UDAF unit Part B } \\
\text { settle plate }\end{array}$ & 1 CFU & 0 CFU & 0 CFU \\
\hline $\begin{array}{l}\text { Isolator Grade A } \\
\text { settle plates }\end{array}$ & 0 CFU & 0 CFU & 0 CFU \\
\hline $\begin{array}{l}\text { Isolator gloves (4x) } \\
\text { glove prints }\end{array}$ & 0 CFU & 0 CFU & 0 CFU \\
\hline $\begin{array}{l}\text { Personnel swabbing } \\
\text { (lab glove) }\end{array}$ & $3 \mathrm{CFU}$ & 4 CFU & 0 CFU \\
\hline
\end{tabular}

The microbiological flora for cleanroom environmental monitoring was classical human commensals and environmental non-sporing rods, no fungi or moulds were detected. The environmental monitoring for room and clean bench parts demonstrates a significantly lower level in actual CFU counts than maximum limits for a Grade $\mathrm{C}$ environment.

For all pre-sterilised tub configurations, it can be seen that a major reduction in contamination, which most likely occurred while touching the transport box for bagged tub handling, is possible without compromising the overall sterile status of the tub and nest. 
Even with the extra challenge to the tub sterility with the controlled but non-classified background environment, the more challenging microbial contamination and deliberately basic gowning, no contamination was detected in the Grade $A$ aseptic processing environment and on the sterile barrier packaging, tub surfaces, nest, and Tyvek lid. Three boxes containing 15 tubs with different configurations (vials, syringes, and cartridges), in total 45 samples, were provided for challenge studies. All showed the same result, providing confidence in the result under atypical worst-case conditions and supporting claims for assured sterility.

From each of the 45 tubs tested, 14 samples (Position 13 - Position 26) were taken under Grade A conditions. All results from the 630 samples taken under Grade A conditions gave the same results of zero cfu recovery; indicating a maintenance of Grade A aseptic conditions and sterility of NTT tubs and containers. This result was achieved without the application of a material disinfection step at GMP grade changes and supports the NTT principles. The reproducibility of the results following $\mathrm{NTT}$ transfers indicates accuracy during the execution of the qualification studies and provides confidence in the results.

\section{Discussion and Conclusion}

The microbial profiling and bioburden study following supply chain delivery of pre-sterilised RTU containers meets acceptance criteria both in terms of study expectation and GMP compliance. After application of the NTT-suitable RTU containers in an NTT process, 0 CFU recovery on sterile barriers, tub-nest components, and Grade A environment was confirmed.

In the case of these challenge studies, the NTT process was undertaken by fully manual double de-bagging (bag end cutting, opening, and contents indexing-ejection without direct contact). In reality, current NTT systems use technology to control manual steps or fully automated bag cutting, opening, and ejection-indexing of contents to the Grade A aseptic process zone without direct contact with the indexing mechanism or secondary bag that remains behind. The first de-bagging step has a lower risk profile and hence allows for less process control but still must meet NTT principles with controlled bag-cutting/opening within a localised unidirectional airflow zone which is at the same Grade as the zone the contents are index-ejected into. NTT is completed without direct contact or need for a material disinfection step. There should be full risk mitigation of human error by following QbD principles for the critical process of pre-sterilised RTU containers tub transfers into the Grade A aseptic processing zone. Fully manual, risk-prone procedures of NTT are not considered GMP in a production setting.

Results of the testing challenge studies performed to verify that the pre-sterilised RTU containers manufactured by Schott in packaging suitable for NTT applications provide supportive data that the NTT process is robust and reproducible; meeting predetermined criteria set for GMP compliance along with current regulatory expectations in following QRM principles for alternative methodologies. 


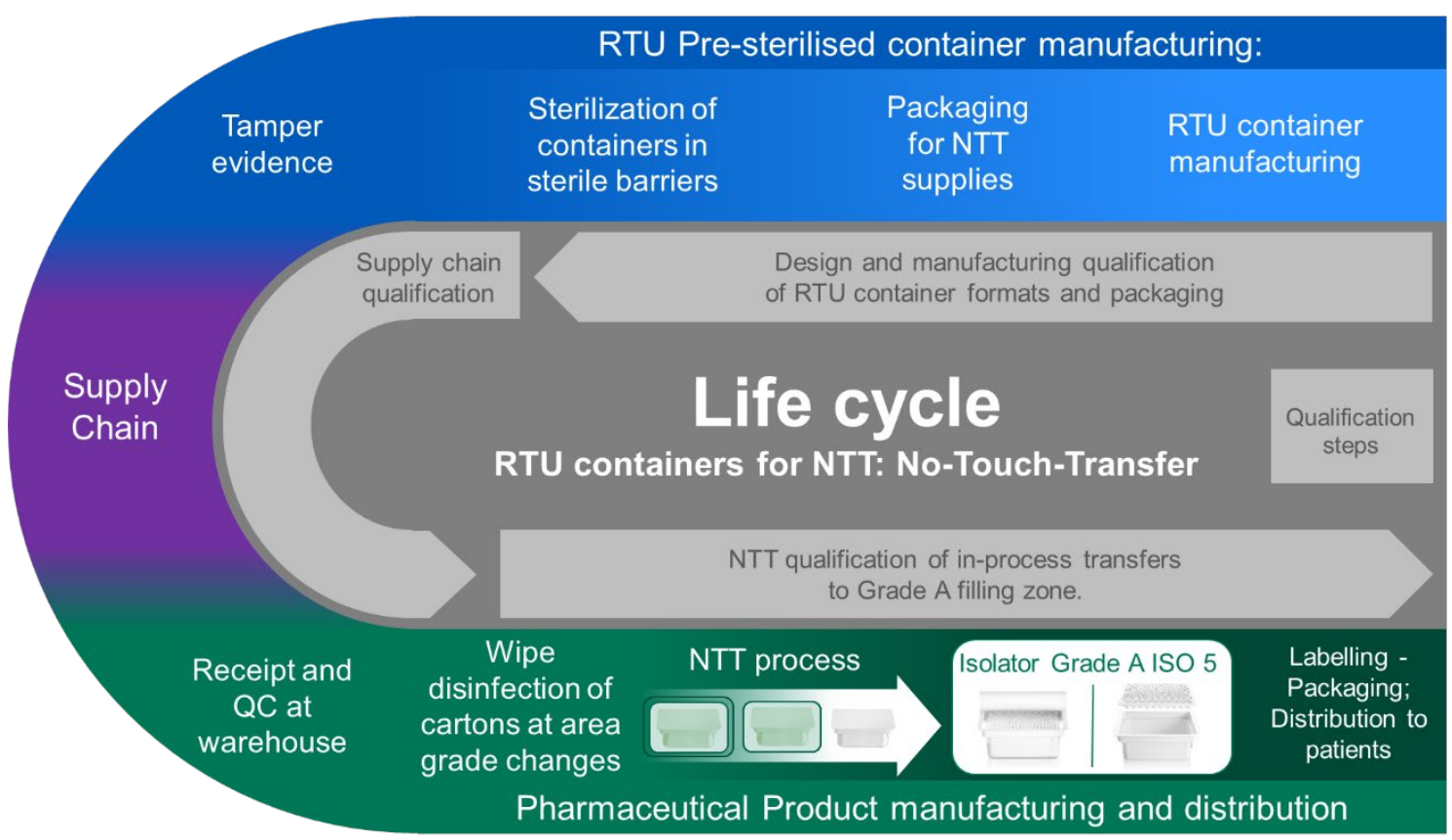

Figure 5: End-to-end qualification of the RTU container life cycle and assurance of sterility: Each step of the process is qualified - from initial sterilization through the supply chain to in-process material transfers to ensure that sterility is maintained through the supply chain and the NTT process.

Regulatory requirements in GMP compliance and regulatory expectations in following QRM that can be justified in a Contamination Control Strategy (CCS) consider that any alternative process should meet or exceed established GMP methods or processes. Study results show NTT meets GMP requirements and follows QRM principles, and thereby, can be justified as a qualified alternative methodology. End-to-end NTT qualification with a double sterile barrier packaging provides assurance of sterility - that the outer tub surfaces (primary sterile barrier), which are sterile in manufacture, remain sterile through the supply chain and that sterility can be maintained with a properly qualified and executed NTT process (Figure 5).

\section{$5 \quad$ References}

01. Drinkwater JL, Novak W, and Cecchetto A. Transfer of ready-to-use sterilised product primary packaging containers and single-use systems into small-batch filling systems in isolators. European Journal of Parenteral and Pharmaceutical Sciences 2015;20(1):23-31.

02. Pharmaceutical and Healthcare Sciences Society Bio-contamination Special Interest Group. Bio-contamination Technical Monograph No. 20: Bio-contamination Characterisation, Control, Monitoring, and Deviation Management in Controlled/GMP Classified Areas, Swindon, UK: PHSS; September 2014.

03. Ljungqvist B, Reinmuller B, Maier C, and Roth A-C. Assessing contamination control of presterilised container tub transfers into an aseptic manufacturing filling isolator via a debagging/no-touchtransfer process step. European Journal of Parenteral and Pharmaceutical Sciences 2016;21(3):90-101. 
04. Drinkwater JL, Morris D, Eaton T, and Hare B. No-touch transfer: pre-sterilised container entry into EU Grade A filling environments following GMP and QRM principles. European Journal of Parenteral and Pharmaceutical Sciences 2018;23(2):43-52.

05. Pharmaceutical and Healthcare Sciences Society Bio-contamination Special Interest Group. Clarity on GMP Guidance Note No.3 Qualification of No-Touch Transfer: NTT for RTU containers. Swindon, UK: PHSS; June 2020.

06. Yu LX, Amidon G, Khan MA, Hoag SW, Polli J, Raju GK, and Woodcock J. Understanding Pharmaceutical Quality by Design. The American Association of Pharmaceutical Scientists Journal 2014;16:771-783.

07. DIN e.V. (Hrsg.) (DIN EN ISO 11607-1:2020-05, 2020): DIN EN ISO 11607-1:2020-05, Packaging for terminally sterilized medical devices - Part 1: Requirements for materials, sterile barrier systems and packaging systems (ISO 11607-1:2019); German version EN ISO 11607-1:2020, Beuth-Verlag, Berlin, 2020.

08. DIN e.V. (Hrsg.) (DIN EN ISO 11607-2:2020-05, 2020): DIN EN ISO 11607-1:2020-05, Packaging for terminally sterilized medical devices - Part 2: Validation requirements for forming, sealing and assembly processes (ISO 11607-2:2019); German version EN ISO 11607-2:2020, Beuth-Verlag, Berlin, 2020.

09. Symonds ID, Martin DL, Davies MC. Facility-based case study: A comparison of the recovery of naturally occurring species of bacteria and fungi on semi-solid media when incubated under standard and dual temperature conditions and its impact on microbial environmental monitoring approach. European Journal of Parenteral and Pharmaceutical Sciences 2016;21(1):7-15.

\section{Acknowledgements}

James L. Drinkwater, Head of PHSS Aseptic Processing special interest group, responsible for introduction of the NTT concept and study management of the proof-of-concept studies. James Drinkwater is also Head of GMP compliance at Franz Ziel GmbH.

Ann-Catherine Roth, Head, responsible for execution of the microbial-bioburden studies at Franz Ziel GmbH Laboratory Services.

Francesca E. Ciraldo, Product Engineer, responsible for the preparation of testing samples at SCHOTT Schweiz AG. 